\title{
Characterization of the Stabilizing PID Controller Region for the Model-Free Time-Delay System
}

\author{
Linlin Ou, ${ }^{1}$ Yuan Su, ${ }^{1}$ and Xuanguang Chen ${ }^{2}$ \\ ${ }^{1}$ College of Information Engineering, Zhejiang University of Technology, Hangzhou 310023, China \\ ${ }^{2}$ Zhuji Sitong Mechanical and Electrical Equipment Manufacturing Co., LTD, Zhuji 311824, China \\ Correspondence should be addressed to Linlin Ou; 1355743770@qq.com
}

Received 25 January 2013; Accepted 21 March 2013

Academic Editor: Mamdouh M. El Kady

Copyright (c) 2013 Linlin Ou et al. This is an open access article distributed under the Creative Commons Attribution License, which permits unrestricted use, distribution, and reproduction in any medium, provided the original work is properly cited.

\begin{abstract}
For model-free time-delay systems, an analytical method is proposed to characterize the stabilizing PID region based on the frequency response data. Such characterization uses linear programming which is computationally efficient. The characteristic parameters of the controller are first extracted from the frequency response data. Subsequently, by employing an extended HermiteBiehler theorem on quasipolynomials, the stabilizing polygon region with respect to the integral and derivative gains $\left(k_{i}\right.$ and $\left.k_{d}\right)$ is described for a given proportional gain $\left(k_{p}\right)$ in term of the frequency response data. Simultaneously, the allowable stabilizing range of $k_{p}$ is derived such that the complete stabilizing set of the PID controller can be obtained easily. The proposed method avoids the complexity and inaccuracy of the model identification and thus provides a convenient approach for the design and tuning of the PID controller in practice. The advantage of the proposed algorithm lies in that the boundaries of the stabilizing region consist of several simple straight lines, the complete stabilizing set can be obtained efficiently, and it can be implemented automatically in computers.
\end{abstract}

\section{Introduction}

Time delay is ubiquitous in many control systems [1]. It may cause the degradation of the control performance and may even render the unstable controlled structure [2,3]. Hence, the problem of time delay has been studied quite extensively in the literature. The closed-loop characteristic equation of time-delayed systems has an infinite number of roots, which makes the analysis and design extremely challenging.

In industry control, the majority of control systems continue to be operated by PID controller despite the recent advances in control theory and implementation. This popularity stems from their structural simplicity and robust performance in a wide range of operating conditions [4]. Accordingly, the design of PID controllers for the time delay processes is of great importance. Conventional approaches to design a PID controller often involve the development of a mathematical model with time delay, that is, transfer function or state space model, which is obtained by using some approximation or simplification. It inevitably causes the identification error between the mathematical model and the corresponding controlled plant. Thus, the control result may not be reliable. Moreover, some variables or parameters are unavailable or difficult to be obtained in practical applications. Another kind of control technique for the systems with time delay is to design a control system by directly using the original input and output data of the controlled plant which can be easily measured experimentally, that is, model-free control design. Most of the modelfree approaches are based on fuzzy logic and neural networks $[5,6]$. On the other hand, model-free approaches received special attention in the area of PID autotuning due to many advantages for tuning PID parameters within a closed-loop setting. Many popular tuning rules such as the ZieglerNichols [7] procedure and the current practice in PID design for process control are based on the frequency response of the plant. Most of the model-free control methods are the single tuning ones; that is to say, they can only satisfy a specified criterion, but not several required design criteria simultaneously. However, in the practical application, most of the industrial controllers are required to satisfy different 
performance criteria simultaneously, either in time domain (e.g., overshoot and settling time) or frequency domain (e.g., phase margin and gain margin). In order to reach this purpose, a natural idea is to first present the stabilizing regions of the PID controller and then determine the PID controller by finding the intersection of the areas of the control parameters meeting each required criterion under the constraint of the resultant stabilizing regions. In the recent years, some effective approaches on determining the stabilizing region of the PID controllers have been reported for the system with time delay based on the transfer function model [8-11]. The approaches in [12] and [13] were developed to determine the entire set of stabilizing first-order/PID controller only based on the frequency response of a given LTI plant. In [14], the D-composition approach is employed to determine the stabilizing PID set for the time-delay plant without the detailed model. Although the graphical method based on the $\mathrm{D}$-composition technique is feasible for the determination of the stabilizing PID region, the characterization of the stability boundaries is very complex and cannot be described by using a simple function, which causes difficulty in the practical application. In addition, the procedure for deriving the stabilizing PID region has to be finished manually and cannot be implemented automatically by the computer.

In this paper, a computationally efficient strategy is proposed to generate the linear programming characterization of the stabilizing PID controllers for the time-delay plant based on frequency response data. Based on the extension of the Hermite-Biehler Theorem, the stabilizing polygon region with respect to $\left(k_{i}, k_{d}\right)$ is derived for a fixed $k_{p}$ value. Moreover, in order to present the entire stabilizing set of the PID controller efficiently, the allowable stabilizing range of $k_{p}$ is also derived on the basis of the frequency response data. The results provide the important approach for the PID controller design satisfying different performance criterion simultaneously only based on the frequency response data of the plant.

The paper is organized as follows. The problem statement is given in Section 2. The method to determine the characteristic parameters necessary for the determination of the stabilizing PID controller is addressed in Section 3. In Section 4, the stabilization of the PID controller for the model-free linear time-delay system is analyzed, and the algorithm of determining the set of all stabilizing sets is proposed. Numerical examples are provided to illustrate the main results in Section 5. Finally, the conclusion is given in Section 6.

\section{Problem Statement}

Consider the feedback configuration with an LTI plant and a PID controller as shown in Figure 1, where $r$ is the reference input, $y$ is the system output, and $C(s)$ is a PID controller with the following form:

$$
C(s)=k_{p}+\frac{k_{i}}{s}+k_{d} s,
$$

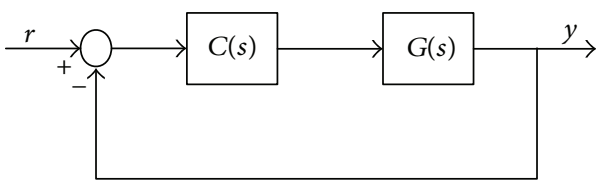

FIGURE 1: Block diagram of the unity feedback control systems.

where $G(s)$ is a single-input single output (SISO) linear timeinvariant (LTI) plant with the frequency response data, and its detailed model is unknown.

The objective of this paper is to directly determine the complete set of the PID controller that can guarantee the system stability based on the frequency response data of the plant, without constructing a state space or transfer function model.

\section{Determination of the Characteristic Parameters}

In this section, the characteristic parameters of the plant that are required to determine the stabilizing set of the PID controller will be presented. Assume that the plant $G(s)$ has the following transfer function:

$$
G(s)=\frac{N(s)}{D(s)} e^{-\theta s},
$$

where $\theta$ is the time delay $N(s)$ and $D(s)$ are polynomials with real coefficients and high degrees $n$ and $m$, respectively. Let $r(N), l(N)$, and $j(N)$ denote the numbers of right-halfplane(RHP), left half-plane(LHP), and imaginary-axis zeros of $G(s)$, respectively, and let $r(D), l(D)$, and $j(D)$ be the numbers of its right half-plane(RHP), left half-plane(LHP), and imaginary-axis poles of $G(s)$, respectively.

The time-delay $\theta$ can be easily determined by imposing the step signal in the input. The lag time between the step signal and the output response signal is $\theta$.

The frequency response of $G(s)$ only is characterized as

$$
G(s)=\frac{N(j \omega)}{D(j \omega)} e^{-j \theta \omega}=G_{r}(\omega)+j G_{i}(\omega),
$$

where $G_{r}(\omega)$ and $G_{i}(\omega)$ are the real and imaginary parts of the frequency response of the plant. For the stable plant, the frequency response data can be obtained in terms of multiple point relay test method in [15]. For the unstable plant, the frequency response data cannot be presented directly. However, we can find a controller $C_{0}(s)$ that can make the closed-loop system stable and then obtain the frequency response data of the plant by measuring the frequency response data of the stable closed-loop system.

The Nyquist and Bode diagrams can be easily sketched based on the frequency response of $G(s)$. From the high frequency slope of the Bode magnitude plot, the relative degree $n-m$ can be presented as follows:

$$
n-m=-\left.\frac{1}{20} \cdot \frac{d P_{d b}(\omega)}{d\left(\log _{10} \omega\right)}\right|_{\omega \rightarrow \infty},
$$


where

$$
P_{d b}(\omega)=20 \log _{10}|G(j \omega)| .
$$

Then, we compute $j(N)$ and $j(D)$. If there exists abrupt increasing change for $T$ times at $\omega=\omega_{t}^{*}(t=1,2,3, \ldots, T)$ in the Bode magnitude plot, $G(s)$ must have the imaginaryaxis poles $s= \pm j \omega_{t}^{*}$. To determine the number of multiple imaginary-axis poles, the plot of $|G(j \omega)| /\left(1-\left(\omega_{t}^{*}\right)^{2}\right)^{u_{t}}$ is drawn for $u_{t}$ increasing from 1 till the abrupt increasing change at $\omega=\omega^{*}$ disappears. Assume that $u_{t}=U_{t}$ when the abrupt increasing change disappears. Thus, we have

$$
j(D)=\sum_{t=1}^{T} 2 U_{t} .
$$

Conversely, If there exists abrupt decreasing change for $T$ times at $\omega=\omega_{t}^{*}(t=1,2,3, \ldots, T)$ in the Bode magnitude plot, $G(s)$ must have the imaginary-axis zeros $s= \pm j \omega_{t}^{*}$. By the similar lines as the computation of $j(D), j(N)$ can also be obtained from the Bode magnitude plot.

Take $l^{*}$ to be a sufficiently large integer. The net change of the phase angle of $G(j \omega)$ as $\omega$ increases from 0 to $2 l^{*} \pi$, denoted as $\Delta_{0}^{2 l^{*} \pi / \theta} \angle G(j \omega)$, can be easily obtained from the phase plot. Moreover, when $l^{*}$ is sufficiently large, the following equation holds

$$
\begin{aligned}
\Delta_{0}^{2 l^{*} \pi / \theta} L G(j \omega)= & -(n-m) \frac{\pi}{2}-[r(N)-r(D)] \pi \\
& -[j(N)-j(D)] \frac{\pi}{2}-2 l^{*} \pi .
\end{aligned}
$$

(1) When the plant is stable, we have $r(D)=0$. Then, from (7), $r(N)$ can be obtained.

(2) When the plant is unstable, the controller $C_{0}(s)$ that can make the closed-loop system stable, is introduced. The closed-loop transfer function is given by

$$
T(s)=\frac{C_{0}(s) G(s)}{1+C_{0}(s) G(s)} .
$$

Transform $T(s)$ into the form

$$
T(s)=\frac{N_{C}(s) N(s)}{e^{\theta s} D_{C}(s) D(s)+N_{C}(s) N(s)},
$$

where $N_{C}(s)$ and $D_{C}(s)$ are the numerator and denominator of the transfer function of $C_{0}(s)$, respectively.

Take the denominator of (9) as

$$
\delta(s)=e^{\theta s} D_{C}(s) D(s)+N_{C}(s) N(s) .
$$

Considering the argument of $T(s)$ in $\left(\left(-2 l^{*} \pi+\eta\right) / \theta,\left(2 l^{*} \pi+\right.\right.$ $\eta) / \theta$ ), where $\eta$ can be chosen in the interval $(0, \pi / 4)$. Due to the stability of the system, all zeros of $\delta(s)$ are in the left halfplane. From the extension of the extended Hermite theorem [11], the net change in phase of $\delta(s)$ in $\left(\left(-2 l^{*} \pi+\eta\right) / \theta,\left(2 l^{*} \pi+\right.\right.$ $\eta) / \theta)$ is $\left(4 l^{*}+n+n_{c}\right) \pi$, where $n_{c}$ is the highest order of $D_{C}(s)$.
Thus, the net change of the phase angle of $T(j \omega)$ as $\omega$ increases from $\left(-2 l^{*} \pi+\eta\right) / \theta$ to $\left(2 l^{*} \pi+\eta\right) / \theta$ is

$$
\begin{aligned}
\Delta_{\left(-2 l^{*} \pi+\eta\right) / \theta}^{\left(2 l^{*} \pi+\theta\right.} & L T(j \omega) \\
= & {[l(N)-r(N)] \pi+\left[l\left(N_{C}\right)-r\left(N_{C}\right)\right] \pi } \\
& -\left(4 l^{*}+n+n_{c}\right) \pi \\
= & -(n-m)-[j(N)+2 r(N)] \\
& -\left[n_{c}-l\left(N_{C}\right)+r\left(N_{C}\right)\right]-4 l^{*}
\end{aligned}
$$

where $r\left(N_{C}\right)$ and $l\left(N_{C}\right)$ denote the numbers of RHP and LHP of the controller $C_{0}(s)$. According to the frequency response data of $T(j \omega), \Delta_{\left.\left(-2 l^{*} \pi\right) / \eta\right) / \theta}^{\left(2 l^{*} \pi+\eta\right)} \angle T(j \omega)$ can be easily derived. The values of $n-m, l\left(N_{C}\right), r\left(N_{C}\right)$, and $j(N)$ have been known. Hence, the value of $r(N)$ can be obtained from (11).

From the frequency response data of $T(j \omega)$, we have

$$
G(j \omega)=\frac{T(j \omega)}{C_{0}(j \omega)[1-T(j \omega)]}
$$

From (12), the argument $\Delta_{\left(-2 l^{*} \pi+\eta\right) / \theta}^{\left(2 l^{*} \pi+\eta\right) / \theta} L G(j \omega)$ is obtained. Furthermore, in terms of (3),

$$
\begin{aligned}
\Delta_{\left(-2 l^{*} \pi+\eta\right) / \theta}^{\left(2 l^{*} \pi+\eta\right) / \theta} L G(j \omega)= & -(n-m) \pi-2[r(N)-r(D)] \pi \\
& -[j(N)-j(D)] \pi-4 l^{*} \pi .
\end{aligned}
$$

Hence, the value of $r(D)$ can be derived from (13).

In the case $n-m=1$,

$$
\left|\frac{a_{n}}{b_{m}}\right|=\lim _{\omega \rightarrow \infty}|G(j \omega) j \omega|
$$

\section{Stabilization Analysis of the PID Controller}

The closed-loop characteristic function of unit feedback system in Figure 1 is

$$
Q(s)=s+\left(k_{i}+k_{p} s+k_{d} s^{2}\right) G(s) .
$$

Multiplying (15) by $e^{\theta s}$, we have

$$
Q(s) e^{\theta s}=s e^{\theta s}+\left(k_{i}+k_{p} s+k_{d} s^{2}\right) G_{0}(s)
$$

where

$$
G_{0}(s)=\frac{N(s)}{D(s)} .
$$


Multiplying (16) by $G_{0}(-s)$, we have

$$
\begin{aligned}
\bar{Q}(s)= & G_{0}(-s) s e^{\theta s}+\left(k_{i}+k_{p} s+k_{d} s^{2}\right) G_{0}(s) G_{0}(-s) \\
=\frac{1}{D(s) D(-s)}[ & s e^{\theta s} D(s) N(-s) \\
& \left.+\left(k_{i}+k_{p} s+k_{d} s^{2}\right) N(s) N(-s)\right] .
\end{aligned}
$$

Substituting $s=j(z / \theta)$ into (18) yields

$$
\begin{gathered}
\bar{Q}_{r}\left(z, k_{i}, k_{d}\right)=\frac{z}{\theta} G_{i}(z)+\left(k_{i}-k_{d} \frac{z^{2}}{\theta^{2}}\right)\left|G\left(j \frac{z}{\theta}\right)\right|^{2}, \\
\bar{Q}_{i}(z)=\frac{z}{\theta}\left[G_{r}(z)+k_{p}\left|G\left(j \frac{z}{\theta}\right)\right|^{2}\right],
\end{gathered}
$$

where $\bar{Q}_{r}\left(z, k_{i}, k_{d}\right)$ and $\bar{Q}_{i}(z)$ are the real and imaginary parts of $\bar{Q}(j(z / \theta))$. The numerator of $Q(s) e^{\theta s}$ in (16) is given by

$$
H(s)=s e^{\theta s} D(s)+\left(k_{i}+k_{p} s+k_{d} s^{2}\right) N(s) .
$$

Substituting $s=j(z / \theta)$ into $H(s)$ yields

$$
H\left(j \frac{z}{\theta}\right)=H_{r}(z)+j H_{i}(z),
$$

where $H_{r}(z)$ and $H_{i}(z)$ represent the real and imaginary parts of $H(j(z / \theta))$. It is seen that $H(s)$ and $Q(s)$ have the same zeros. Substituting $s=j(z / \theta)$ into $H(s)$ and multiplying it by $N(-j(z / \theta))$ yields

$$
H\left(j \frac{z}{\theta}, k_{p}, k_{i}, k_{d}\right) N\left(-j \frac{z}{\theta}\right)=p\left(z, k_{i}, k_{d}\right)+j q\left(z, k_{p}\right),
$$

where

$$
\begin{aligned}
& p\left(k_{i}, k_{d}\right)= p_{1}(z)+\left(k_{i}-\frac{k_{d} z^{2}}{\theta^{2}}\right)\left[N_{r}^{2}(z)+N_{i}^{2}(z)\right] \\
& q\left(z, k_{p}\right)=\frac{z}{\theta}\left\{q_{1}(z)+k_{p}\left[N_{r}^{2}(z)+N_{i}^{2}(z)\right]\right\} \\
& p_{1}(z)=-\frac{z}{\theta}\left[D_{i}(z) N_{r}(z)-D_{r}(z) N_{i}(z)\right] \cos (z) \\
&-\frac{z}{\theta}\left[D_{r}(z) N_{r}(z)+D_{i}(z) N_{i}(z)\right] \sin (z) \\
& q_{1}(z)=\left[D_{r}(z) N_{r}(z)+D_{i}(z) N_{i}(z)\right] \cos (z) \\
&-\left[D_{i}(z) N_{r}(z)-D_{r}(z) N_{i}(z)\right] \sin (z)
\end{aligned}
$$

Actually, $H\left(j(z / \theta), k_{p}, k_{i}, k_{d}\right) N(-j(z / \theta))$ in (23) is the numerator of (18).

Lemma 1. If all zeros of $H(s)$ lie in the left half-plane, the net change of the phase angle of $H(j \omega)$ for $\omega$ increasing from $-2 l^{*} \pi+\eta$ to $2 l^{*} \pi+\eta$ satisfies the following:

$$
\Delta_{\left(-2 l^{*} \pi+\eta\right)}^{\left(2 l^{*} \pi+\eta\right)} \angle H\left(j \frac{z}{\theta}\right)=\left(4 l^{*}+n+1\right) \pi .
$$

Proof. In terms of the results in [11], it is known that if all zeros of $H(s)$ and $Q(s)$ lie in the left half-plane, $H_{r}(\omega)$ and $H_{i}(\omega)$ have exactly $4 l^{*}+n+1$ real zeros in the interval $\left(-2 l^{*} \pi+\eta, 2 l^{*} \pi+\eta\right)$, and the zeros of $H_{r}(\omega)$ and $H_{i}(\omega)$ interlace. Denote the minimal and maximal zeros of $H_{i}(\omega)$ in $\left(-2 l^{*} \pi+\eta, 2 l^{*} \pi+\eta\right)$ as $z_{\min }$ and $z_{\max }$. It can be derived that

$$
\Delta_{z_{\min }}^{z_{\max }} L H\left(j \frac{z}{\theta}\right)=\left(4 l^{*}+n+1\right) \pi
$$

As $z \rightarrow+\infty$, we have

$$
\begin{gathered}
H_{r}(z)= \begin{cases}-\frac{z}{\theta} a_{n}(j)^{n} z^{n} \sin (z) & \text { for } n \text { even } \\
-\frac{z}{\theta} a_{n}(j)^{n-1} z^{n} \cos (z) & \text { for } n \text { odd }\end{cases} \\
H_{i}(z)= \begin{cases}\frac{z}{\theta} a_{n}(j)^{n} z^{n} \cos (z) & \text { for } n \text { even } \\
-\frac{z}{\theta} a_{n}(j)^{n-1} z^{n} \sin (z) & \text { for } n \text { odd }\end{cases}
\end{gathered}
$$

where $a_{n}$ is the coefficient of the highest term of $D(s)$. In the case of $n$ even, from (31), we have

$$
\begin{aligned}
& \Delta_{-2 l^{*} \pi+\eta}^{z_{\min }} \angle H\left(j \frac{z}{\theta}\right)=\frac{\pi}{2}-\eta, \\
& \Delta_{z_{\max }}^{2 l^{*} \pi+\eta} \angle H\left(j \frac{z}{\theta}\right)=\frac{\pi}{2}+\eta .
\end{aligned}
$$

Thus, we have

$$
\begin{aligned}
\Delta_{-2 l^{*} \pi+\eta}^{2 l^{*} \pi+\eta} L H\left(j \frac{z}{\theta}\right)= & \Delta_{-2 l^{*} \pi+\eta}^{z_{\min }} \angle H\left(j \frac{z}{\theta}\right)+\Delta_{z_{\min }}^{z_{\max }} \angle H\left(j \frac{z}{\theta}\right) \\
& +\Delta_{z_{\max }}^{2 l^{*} \pi+\eta} \angle H\left(j \frac{z}{\theta}\right) \\
= & \left(4 l^{*}+n+1\right) \pi .
\end{aligned}
$$

In the case of $n$ odd, from (31), we have

$$
\begin{gathered}
\Delta_{-2 l^{*} \pi+\eta}^{z_{\min }} L H\left(j \frac{z}{\theta}\right)=\pi-\eta, \\
\Delta_{z_{\max }}^{2 l^{*} \pi+\eta} \angle H\left(j \frac{z}{\theta}\right)=\eta .
\end{gathered}
$$

Thus, (28) also holds for $n$ odd. 
When $z \rightarrow \infty$, we have

$$
\begin{gathered}
p(z)=\left\{\begin{array}{l}
-\frac{z}{\theta}\left[a_{n} b_{m}(j)^{n+m} z^{n+m} \sin (z)+O\left(z^{n+m-1}\right)\right] \\
-\frac{z}{\theta}\left[a_{n} b_{m}(j)^{n+m} z^{n+m-1} \cos (z)+O\left(z^{n+m-1}\right)\right] \\
-\frac{z}{\theta}\left[-a_{n} b_{m}(j)^{n+m} z^{n+m-1} \cos (z)+O\left(z^{n+m-1}\right)\right] \\
\text { for } n \text { even and } m \text { odd }
\end{array}\right. \\
q(z)=\left\{\begin{array}{lr}
\frac{z}{\theta}\left[a_{n} b_{m}(j)^{n+m} z^{n+m} \cos (z)+O\left(z^{n+m-1}\right)\right] \\
\frac{z}{\theta}\left[-a_{n} b_{m}(j)^{n+m-1} z^{n+m} \sin (z)+O\left(z^{n+m-1}\right)\right] \\
\frac{z}{\theta}\left[a_{n} b_{m}(j)^{n+m-1} z^{n+m} \sin (z)+O\left(z^{n+m-1}\right)\right] \\
\text { for } n \text { even and } m \text { odd },
\end{array}\right.
\end{gathered}
$$

where $b_{m}$ is the coefficient of the highest term of $N(s)$.

Definition 2. Take $0=z_{0}<z_{1}<z_{2}<\cdots<z_{d-1}$ to be the real and distinct zeros of $\bar{Q}_{i}$ in $\left(0,2 l^{*} \pi+\eta\right)$. These roots are also the roots of $q(z)$ in $\left(-2 l^{*} \pi+\eta, 2 l^{*} \pi+\eta\right)$. Define $i_{0}, i_{1}, i_{2}, \ldots, i_{d}$ as follows.

(1) If the plant has the imaginary-axis zero at $z_{t} / \theta$, then define $i_{t}=0$.

(2) If the plant has a zero at the origin, then define $i_{0}=$ $\operatorname{sgn}\left(d\left[\bar{Q}_{i}(z)\right] /\left.d z\right|_{z=0}\right)$, where $\bar{Q}_{i}(z)$ is given in (20).

(3) For all other $t=0,1,2, \ldots d, i_{t}=1$ or -1 .

Definition 3. Let $I=\left\{i_{0}, i_{1}, \ldots\right\}$. The signature $\gamma(I)$ is denoted by

$$
\begin{aligned}
& \gamma(I)
\end{aligned}
$$

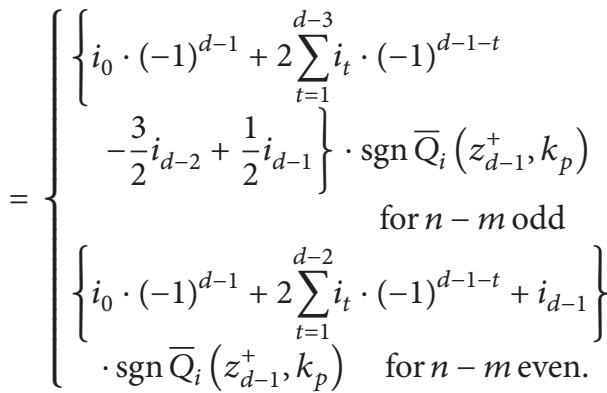

Theorem 4. The necessary and sufficient conditions for the stability of closed-loop system are that there exists $I=$ $\left\{i_{0}, i_{1}, \ldots\right\}$ such that

$$
\gamma(I)=4 l^{*}+(n-m)+j(N)+2 r(N),
$$

where $i_{t}=\operatorname{sgn}\left[\bar{Q}_{r}\left(z_{c-1}, k_{i}, k_{d}\right)\right]$, and let $0=z_{0}<z_{1}<z_{2}<$ $\cdots<z_{d-1}$ be the real and distinct zeros of $\bar{Q}_{i}$ in $\left(0,2 l^{*} \pi+\eta\right)$. For a fixed $k_{p}$, if there exists one string $I=\left\{i_{0}, i_{1}, \ldots\right\}$ satisfying
(38), the stabilizing set of $\left(k_{d}, k_{i}\right)$ is the intersection of the following inequalities:

$$
\left[\left(k_{i}-k_{d} \frac{z^{2}}{\theta^{2}}\right)\left|G\left(j \frac{z}{\theta}\right)\right|^{2}+\frac{z}{\theta} G_{i}(z)\right] i_{t}>0 .
$$

In addition, for a given LTI time-delay plant with $n-m=1$, the stabilizing values of $k_{d}$ have to satisfy another condition $-\left|a_{n} / b_{m}\right|<k_{d}<1 /\left|a_{n} / b_{m}\right|$.

Proof. If $z_{t}, z_{t+1}$ are the roots of $q(z)$, then the net change from $z_{t}$ to $z_{t+1}$ can be described as

$$
\begin{array}{rl}
\Delta_{z_{t}}^{z_{t+1}} & L \bar{Q}\left(j \frac{z}{\theta}\right) \\
\quad= & \frac{1}{2} \pi\left[\operatorname{sgn}\left[q\left(z_{t}\right)\right]-\operatorname{sgn}\left[q\left(z_{t+1}\right)\right]\right] \cdot \operatorname{sgn}\left[p\left(z_{t}^{+}\right)\right],
\end{array}
$$

where

$$
\begin{gathered}
\operatorname{sgn}\left[q\left(z_{t+1}^{+}\right)\right]=-\operatorname{sgn}\left[q\left(z_{t}^{+}\right)\right] \\
\operatorname{sgn}\left[q\left(z_{t}^{+}\right)\right]=(-1)^{d-t-1} \operatorname{sgn}\left[q\left(z_{d-1}^{+}\right)\right] .
\end{gathered}
$$

Take $Z_{0}<Z_{1}<Z_{2}<\cdots<Z_{c-1}$ as the roots of $\bar{Q}_{i}(z)$ in $\left(-2 l^{*} \pi+\eta, 2 l^{*} \pi+\eta\right)$. It is obtained that

$$
\begin{aligned}
& \Delta_{Z_{0}}^{Z_{c-1}} L \bar{Q}\left(j \frac{z}{\theta}\right) \\
&=\frac{\pi}{2}\left\{\operatorname{sgn}\left[p\left(Z_{0}\right)\right] \cdot(-1)^{c-1}+2 \sum_{t=1}^{c-2} \operatorname{sgn}\left[p\left(Z_{t}\right)\right]\right. \\
&\left.\cdot(-1)^{c-1-t}+\operatorname{sgn}\left[p\left(Z_{c-1}\right)\right]\right\} \cdot \operatorname{sgn}\left[q\left(Z_{c-1}^{+}\right)\right] .
\end{aligned}
$$

Since

$$
\Delta_{-2 l^{*} \pi+\eta}^{Z_{0}} L \bar{Q}\left(j \frac{z}{\theta}\right)+\Delta_{Z_{c-1}}^{2 *^{*} \pi+\eta} L \bar{Q}\left(j \frac{z}{\theta}\right)=\pi,
$$

we have

$$
\begin{aligned}
& \Delta_{-2 l^{*} \pi+\eta}^{2 l^{*} \pi+\eta} L \bar{Q}\left(j \frac{z}{\theta}\right) \\
&=\frac{\pi}{2}\{ \operatorname{sgn}\left[p\left(Z_{0}\right)\right] \cdot(-1)^{c-1}+2 \sum_{t=1}^{c-2} \operatorname{sgn}\left[p\left(Z_{t}\right)\right] \\
&\left.\cdot(-1)^{c-1-t}+\operatorname{sgn}\left[p\left(Z_{c-1}\right)\right]\right\} \cdot \operatorname{sgn}\left[q\left(Z_{c-1}^{+}\right)\right]+\pi .
\end{aligned}
$$

It can be obtained that

$$
\begin{aligned}
& \Delta_{-2 l^{*} \pi+\eta}^{2 l^{*} \pi+\eta} L \bar{Q}\left(j \frac{z}{\theta}\right) \\
& \quad=\Delta_{-2 l^{*} \pi+\eta}^{2 l^{*} \pi+\eta} L H\left(j \frac{z}{\theta}\right)-\pi[l(N)-r(N)] .
\end{aligned}
$$


In terms of Lemma 1, if all the zeros of $H(s)(Q(s))$ lie in the left-half plane, then

$$
\Delta_{\left(-2 l^{*} \pi+\eta\right)}^{\left(2 l^{*} \pi+\eta\right)} L H\left(j \frac{z}{\theta}\right)=\left(4 l^{*}+n+1\right) \pi .
$$

It follows that

$$
\begin{aligned}
& \Delta_{-2 l^{*} \pi+\eta}^{2 l^{*} \pi+\eta} L \bar{Q}\left(j \frac{z}{\theta}\right) \\
& \quad=\left(4 l^{*}+n+1\right) \pi-\pi[m-j(N)-2 r(N)] .
\end{aligned}
$$

Substituting (44) into (47) yields

$$
\begin{aligned}
& \left\{\operatorname{sgn}\left[p\left(Z_{0}\right)\right] \cdot(-1)^{c-1}+2 \sum_{t=1}^{c-2} \operatorname{sgn}\left[p\left(Z_{t}\right)\right] \cdot(-1)^{c-1-t}\right. \\
& \left.+\operatorname{sgn}\left[p\left(Z_{c-1}\right)\right]\right\} \cdot \operatorname{sgn}\left[q\left(Z_{c-1}^{+}\right)\right] \\
& \quad=2\left(4 l^{*}+n\right)-2[l(N)-r(N)]
\end{aligned}
$$

From (24), $q\left(z, k_{p}\right)$ is the odd function, and it has the same roots in $\left(-2 l^{*} \pi+\eta, 0\right)$ and $\left(0,2 l^{*} \pi-\eta\right)$. Furthermore, from (35), $q\left(z, k_{p}\right)$ has no real zero in $\left(2 l^{*} \pi-\eta, 2 l^{*} \pi+\eta\right)$ when $m+n$, even and it has one real zero in $\left(2 l^{*} \pi-\eta, 2 l^{*} \pi+\eta\right)$ when $m+n$ odd. Hence, by considering the roots of $q\left(z, k_{p}\right)$ in the interval $\left[0,2 l^{*} \pi+\eta\right)$. It is easily known that

$$
D\left(j \frac{z}{\theta}\right) D\left(-j \frac{z}{\theta}\right)=D_{r}^{2}(z)+D_{i}^{2}(z)>0 .
$$

Thus, the expression (48) can be rewritten as

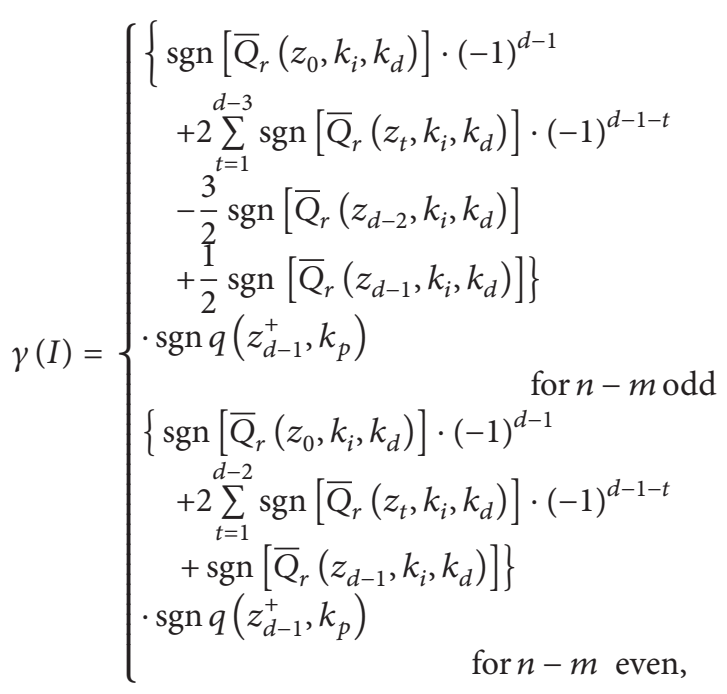

where $\gamma(I)=4 l^{*}+(n-m)+j(N)+2 r(N)$. By taking $i_{t}=$ $\operatorname{sgn}\left[\bar{Q}_{r}\left(z_{c-1}, k_{i}, k_{d}\right)\right]$, the expression (37) can be obtained. This completes the proof.

The following theorem is given to determine the allowable stabilizing range of $k_{p}$.
Theorem 5. The necessary condition for the $k_{p}$ value leading to the existence of the stabilizing $\left(k_{d}, k_{i}\right)$ region is that

$$
k_{p}=-\frac{G_{r}(z)}{|G(j(z / \theta))|^{2}}
$$

has at least $f$ distinct roots in $\left(0,2 l^{*} \pi+\eta\right)$, where

$$
f \geq\left\{\begin{array}{l}
2 l^{*}+\frac{(n-m)-j(N)}{2}+r(N)+\frac{1}{2} \\
\text { for } n-m \text { odd } \\
2 l^{*}+\frac{(n-m)-j(N)}{2}+r(N) \\
\text { for } n-m \text { even } .
\end{array}\right.
$$

By Combining Theorem 4 and Definition 3, the proof of Theorem 5 can be easily proved. Thus, its proof procedure is omitted here.

Based on the results presented in Theorems 4 and 5, the algorithm to determine the stabilizing sets of PID controller based on the frequency response data of the plant is given as follows.

Step 1. Determine the characteristic parameters $n-m, \theta, l(N)$, $l(D), r(N), r(D)$, and $\left|a_{n} / b_{m}\right|$.

Step 2. Present the curve of $|G(j(z / \theta))|, G_{r}(z)$ and $G_{i}(z)$ with respect to $z$ based on the frequency response data of $G(j \omega)$, where $z=\theta \omega$.

Step 3. Choose the proper values of $\eta$ and $l^{*}$.

Step 4. Compute the allowable stabilizing range of $k_{p}$ based on Theorem 5.

Step 5. For a grid point of $k_{p}$, compute the real and distinct zeros of $\bar{Q}_{i}(z)$ in (20) in the interval $\left[0,2 l^{*} \pi+\eta\right)$ and denote them by $z_{0}, z_{1}, z_{2}, \ldots z_{d-1}$ in the increasing order.

Step 6. According to Theorem 4 and Definition 3, find $I$ satisfying (38).

Step 7. Present the stabilizing set of $\left(k_{d}, k_{i}\right)$ by computing the intersection of the inequalities determined by (39) with respect to $I$.

Step 8. Go to Step 5 with another grid point of $k_{p}$ till all the grid points are considered.

\section{Numerical Study}

Example 6. First, we consider the plant in [11]. Collecting the plant frequency response data, the Nyquist plot and the Bode diagrams of the plant are shown in Figures 2 and 3, respectively.

According to Section 3, the characteristic parameters of the plant are firstly obtained. From the Bode magnitude plot in Figure 3, we have

$$
n-m=2 \text {. }
$$




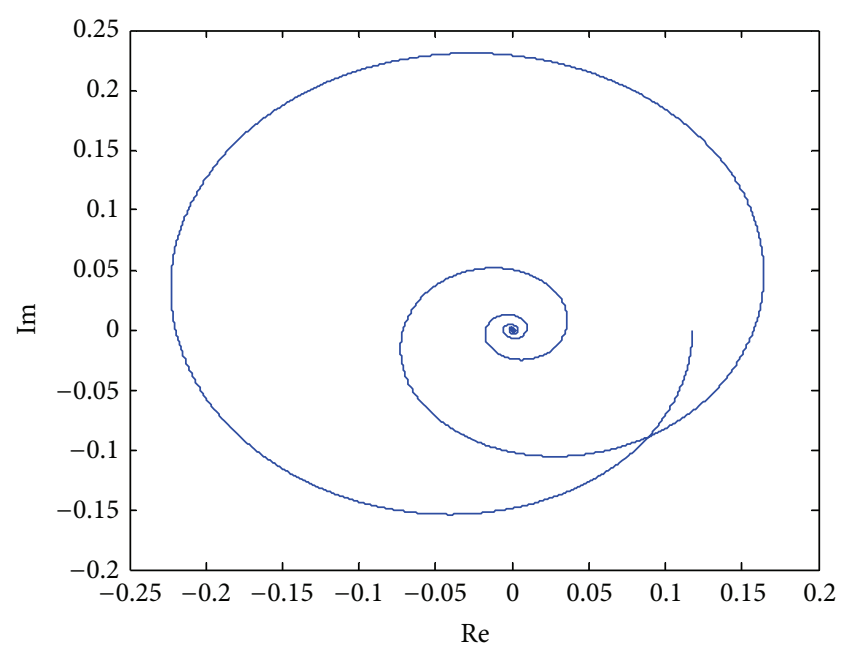

Figure 2: The Nyquist plot for Example 6.

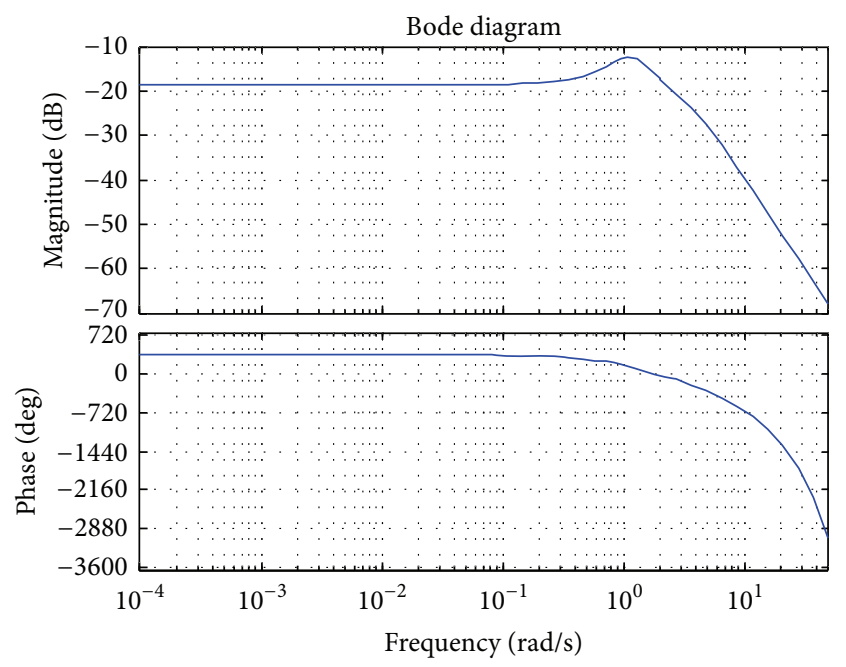

Figure 3: The Bode diagram for Example 6.

Let $l^{*}=8$, and the net change of the phase is depicted in bode plot as follows:

$$
\Delta_{0}^{16 \pi} \angle G\left(j \frac{z}{\theta}\right)=-3360 \approx-19 \pi
$$

Since the plant is stable, from the following equation:

$$
\begin{aligned}
& \Delta_{0}^{2 l^{*} \pi} \angle G\left(j \frac{z}{\theta}\right) \\
& \quad=-(n-m) \cdot \frac{\pi}{2}-2[r(N)-r(D)] \cdot \frac{\pi}{2}-2 l^{*} \pi .
\end{aligned}
$$

It follows that $r(N)=2$.

Choose $\eta=0.1$ and $l^{*}=4$ to find the allowable stabilizing range of $k_{p}$. It is seen from Theorem 5 that the following equation must have at least 11 zeros in $(0,25.233)$ as follows:

$$
k_{p}=-\frac{G_{r}(z)}{|G(j(z / \theta))|^{2}} .
$$

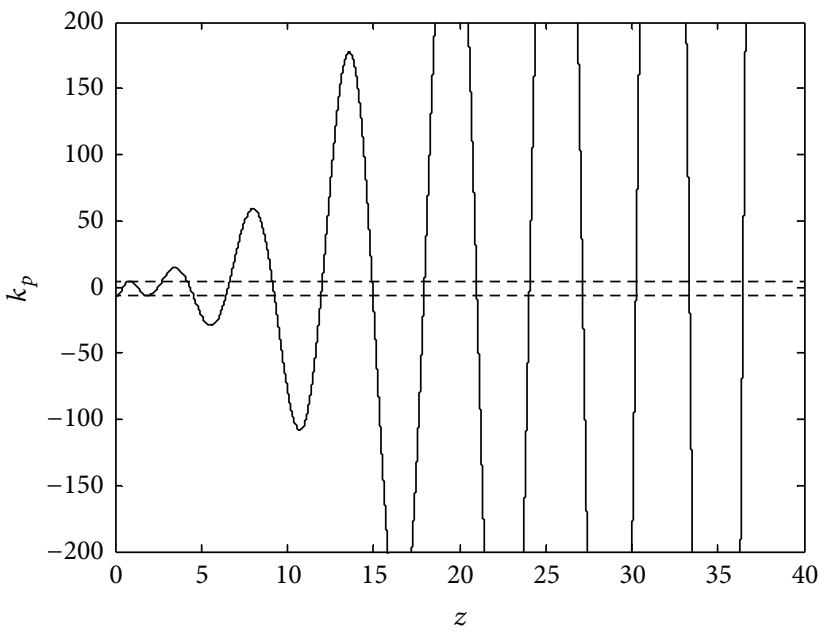

FIGURE 4: Graph of the function $k_{p}$.

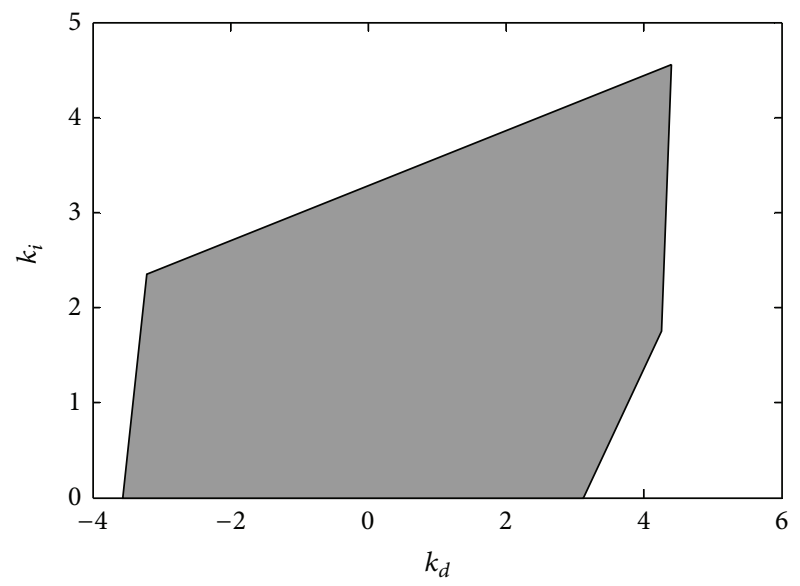

FIGURE 5: Stabilizing range of $\left(k_{i}, k_{d}\right)$ when $k_{p}=1$.

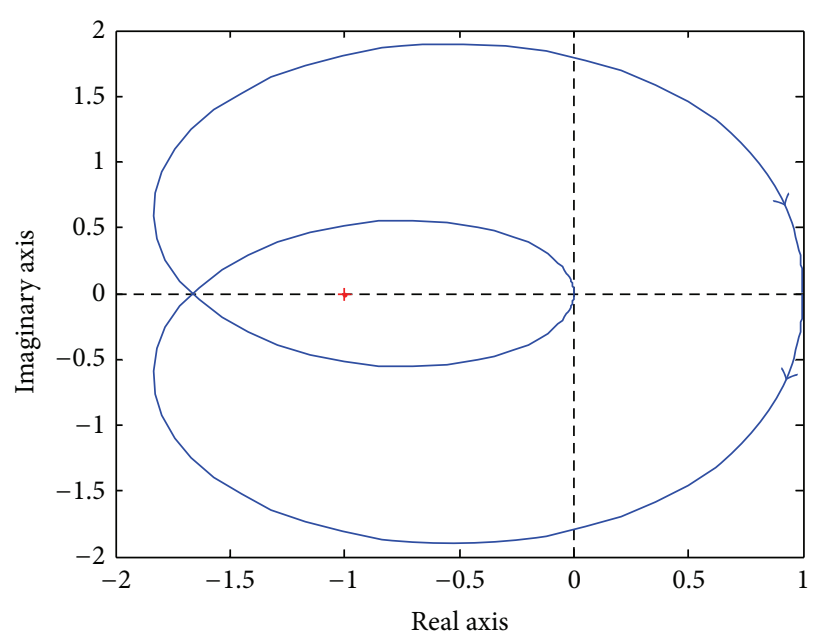

Figure 6: The Nyquist plot for Example 7. 


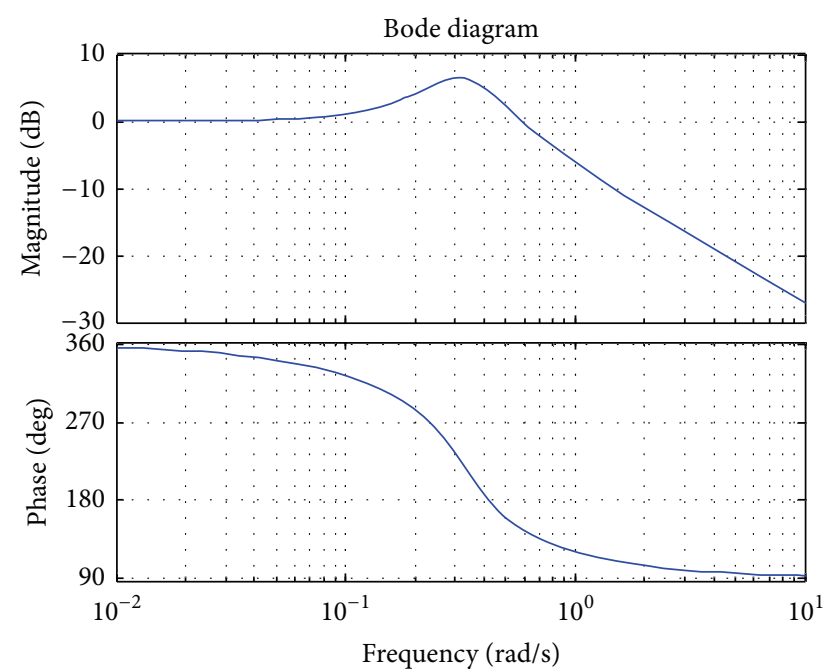

Figure 7: The Bode diagram for Example 7.

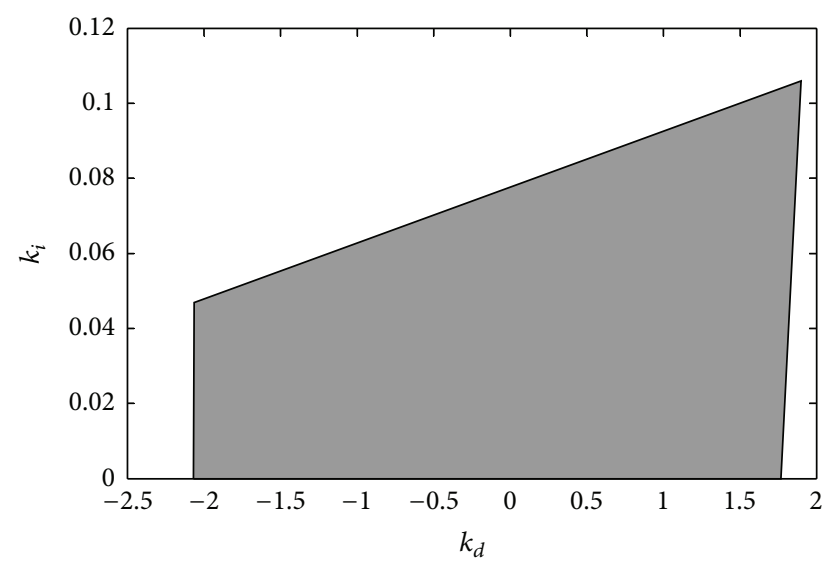

FIGURE 8: Stabilizing range of $\left(k_{i}, k_{d}\right)$ when $k_{p}=1$.

By the plot in Figure 4, it is easy to verify that such a condition holds within the interval $-6.611<k_{p}<4.633$. Thus, the allowable stabilizing range of $k_{p}$ can be obtained. are

Choose $k_{p}=1$ within $(-6.611,4.633)$. The zeros of $\bar{Q}_{i}(z)$

$$
\begin{gathered}
z_{0}=0, \quad z_{1}=0.522, \quad z_{2}=1.24, \quad z_{3}=2.574, \\
z_{4}=4.31, \quad z_{5}=6.60, \quad z_{6}=9.17, \quad z_{7}=12.01, \\
z_{8}=14.95, \quad z_{9}=17.96, \quad z_{10}=20.99, \quad z_{11}=24.01 .
\end{gathered}
$$

From Theorem 4, we have

$$
\begin{gathered}
\left(-i_{0}+2 i_{1}-2 i_{2}+2 i_{3}-2 i_{4}+2 i_{5}-2 i_{6}+2 i_{7}-2 i_{8}\right. \\
\left.+2 i_{9}-2 i_{10}+i_{11}\right) \cdot \operatorname{sgn} \bar{Q}_{i}\left(z_{11}^{+}, k_{p}\right)=22 .
\end{gathered}
$$

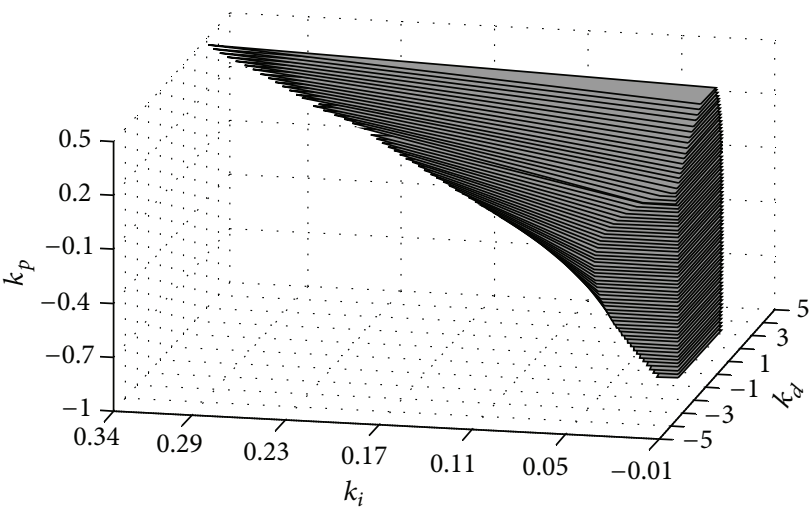

FIgURE 9: Complete stabilizing set of the PID controller.

Thus, the corresponding stings $I$ is

$$
\begin{aligned}
& \left\{i_{0}, i_{1}, i_{2}, i_{3}, i_{4}, i_{5}, i_{6}, i_{7}, i_{8}, i_{9}, i_{10}, i_{11}\right\} \\
& \quad=\{1,-1,1,-1,1,-1,1,-1,1,-1,1,-1\} .
\end{aligned}
$$

By using (39), it is concluded that the stabilizing $\left(k_{i}, k_{d}\right)$ corresponding to $k_{p}=1$ have to simultaneously satisfy the following:

$$
\begin{gathered}
k_{i}>0, \quad k_{i}<0.2725 k_{d}+4, \\
k_{i}>1.5376 k_{d}-8, \quad k_{i}<6.6255 k_{d}+21, \\
k_{i}>18.5761 k_{d}-71, \\
k_{i}<43.56 k_{d}+258, \quad k_{i}>84.0889 k_{d}-720, \\
k_{i}<144.2401 k_{d}+1658, \quad k_{i}>223.5025 k_{d}-3245, \\
k_{i}<322.5616 k_{d}+5674, \quad k_{i}>440.5801 k_{d}-9107, \\
k_{i}<576.4801 k_{d}+13360 .
\end{gathered}
$$

From these inequalities, the stabilizing region of $\left(k_{i}, k_{d}\right)$ is sketched in Figure 5. The complete stabilizing set of the PID controller can also be derived by sweeping over the allowable stabilizing range of $k_{p}$.

Example 7. Let us consider the plant only with the frequency response data, whose Nyquist plot and Bode diagrams are shown in Figures 6 and 7, respectively.

Following the same lines as Example 6, it can be obtained, that $n-m=1$ and $r(N)=1$. Let $\eta=0.1$, and let $l^{*}=4$. From Theorem 5, it is derived that the feasible range of $k_{p}$ is $(-1,4.6)$. For $k_{p}=-0.5$ within the resultant range, the stabilizing region of $\left(k_{i}, k_{d}\right)$ is shown in Figure 8 . By sweeping over the $k_{p}$ values in the interval $(-1,4.6)$, the complete stabilizing set of the PID controller is presented, which is shown in Figure 9.

\section{Conclusion}

In this paper, we have proposed an analytical method to characterize the stabilizing PID region for a linear time-delay 
plant without parametric model using linear programming. Several characteristic parameters, which are required for the determination of the stabilizing PID controller, were firstly provided by using the frequency response information. By employing an extended Hermite-Biehler theorem applicable to quasipolynomials, the $2 \mathrm{D}$ parametric region in the $\left(k_{i}, k_{d}\right)$ space was analytically developed, and its boundaries consist of only several straight lines. Meanwhile, the allowable stabilizing range of $k_{p}$ is developed. Thus, the $3 \mathrm{D}$ visualization of all the stabilizing PID controllers can be conveniently presented only based on the frequency response data of the controlled plant. The results are applicable to arbitrary SISO linear time-delay system, including stable and unstable plants, even the plants with imaginary zeros or poles. The proposed algorithm can be extended to carry out the optimal design of the PID controllers and the design of the PID controllers that can satisfy various performance indices without detailed models.

\section{Acknowledgments}

This paper is supported by the National Science Foundation of China (61273116), National Science Funds of Zhejiang Province (Y1111012), and the National Nature Science Funds for Distinguished Young Scholar under Grant (61025016).

\section{References}

[1] K. Gu, V. Kharitonov, and J. Chen, Stability of Time-Delay Systems, Birkhauser, Boston, Mass, USA, 2003.

[2] Y. X. Qing, Y. Q. Liu, and L. Wang, Stabilization of Dynamic System with Time-Delay, Academic Science, Beijing, China, 1989.

[3] L. Xie, E. Fridman, and U. Shaked, "Robust $\mathrm{H}_{\infty}$ control of distributed delay systems with application to combustion control," IEEE Transactions on Automatic Control, vol. 46, no. 12, pp. 1930-1935, 2001.

[4] W. L. Zang, Y. G. Wang, Z. Guo, and Y. X. Wang, "Satisfactory PID design for servo systems based on iterative LMI technique," Control Theory and Applications, vol. 23, no. 6, pp. 967-975, 2006.

[5] S. Bernt, "Model-free tracking of cars and people based on color regions," Image and Vision Computing, vol. 24, no. 11, pp. 11721178, 2006.

[6] Q. D. Qing and R. J. Li, "Particle swarm optimization algorithm mimicking biological ideal free distribution model," Control and Decision, vol. 26, no. 12, pp. 46-51, 2011.

[7] J. Z. Ziegler, N. B. Nichols, and N. Y. Rochester, "Optimum settings for automatic controllers," Transactions of the ASME, vol. 64 , pp. $759-768,1942$.

[8] A. Datta, M. T. Ho, and S. P. Bhattacharyya, Structure and Synthesis of PID Controllers, Springer, London, UK, 2000.

[9] M. T. Söylemez, N. Munro, and H. Baki, "Fast calculation of stabilizing PID controllers," Automatica, vol. 39, no. 1, pp. 121126, 2003.

[10] D. Ackermann and D. Kaesbauer, "Stable polyhedra in parameter space," Automatica, vol. 39, no. 5, pp. 937-943, 2003.

[11] L. L. Ou, W. D. Zhang, and L. Yu, "Low-order stabilization of LTI systems with time delay," IEEE Transactions on Automatic Control, vol. 54, no. 4, pp. 774-787, 2009.
[12] L. H. Keel and S. P. Bhattacharyya, "PID controller synthesis free of analytical models," in Proceedings of the 16th Triennial World Congress of International Federation of Automatic Control (IFAC '05), pp. 367-372, Prague, Czech Republic, July 2005.

[13] L. H. Keel and S. P. Bhattacharyya, "Direct synthesis of first order controllers from frequency response measurements," in Proceedings of the American Control Conference (ACC '05), pp. 1192-1196, Portland, Ore, USA, June 2005.

[14] Y. Li, A. Sheng, and Y. Wang, "Synthesis of PID-type controllers without parametric models: a graphical approach," Energy Conversion and Management, vol. 49, no. 8, pp. 2392-2402, 2008.

[15] Q. G. Wang, C. C. Hang, and Q. Bi, "A technique for frequency response identification from relay feedback," IEEE Transactions on Control Systems Technology, vol. 7, no. 1, pp. 122-128, 1999. 


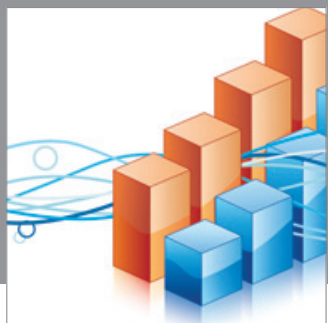

Advances in

Operations Research

mansans

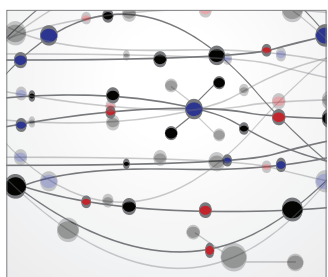

The Scientific World Journal
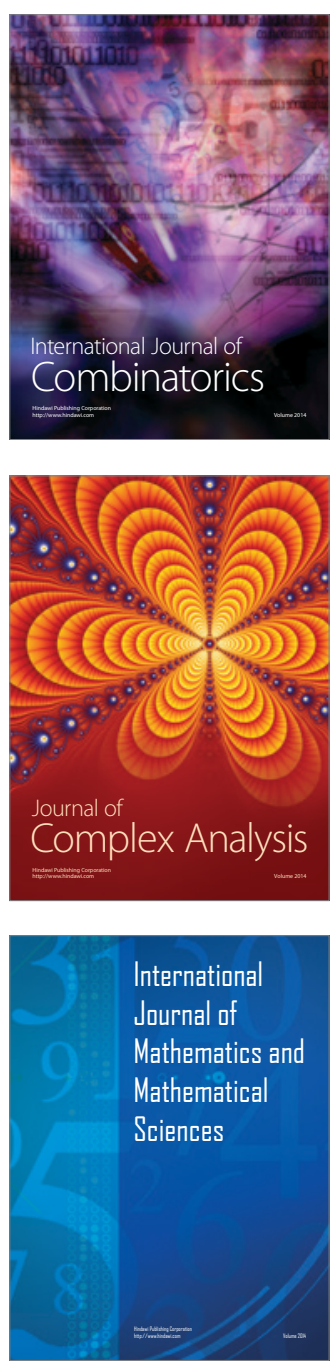
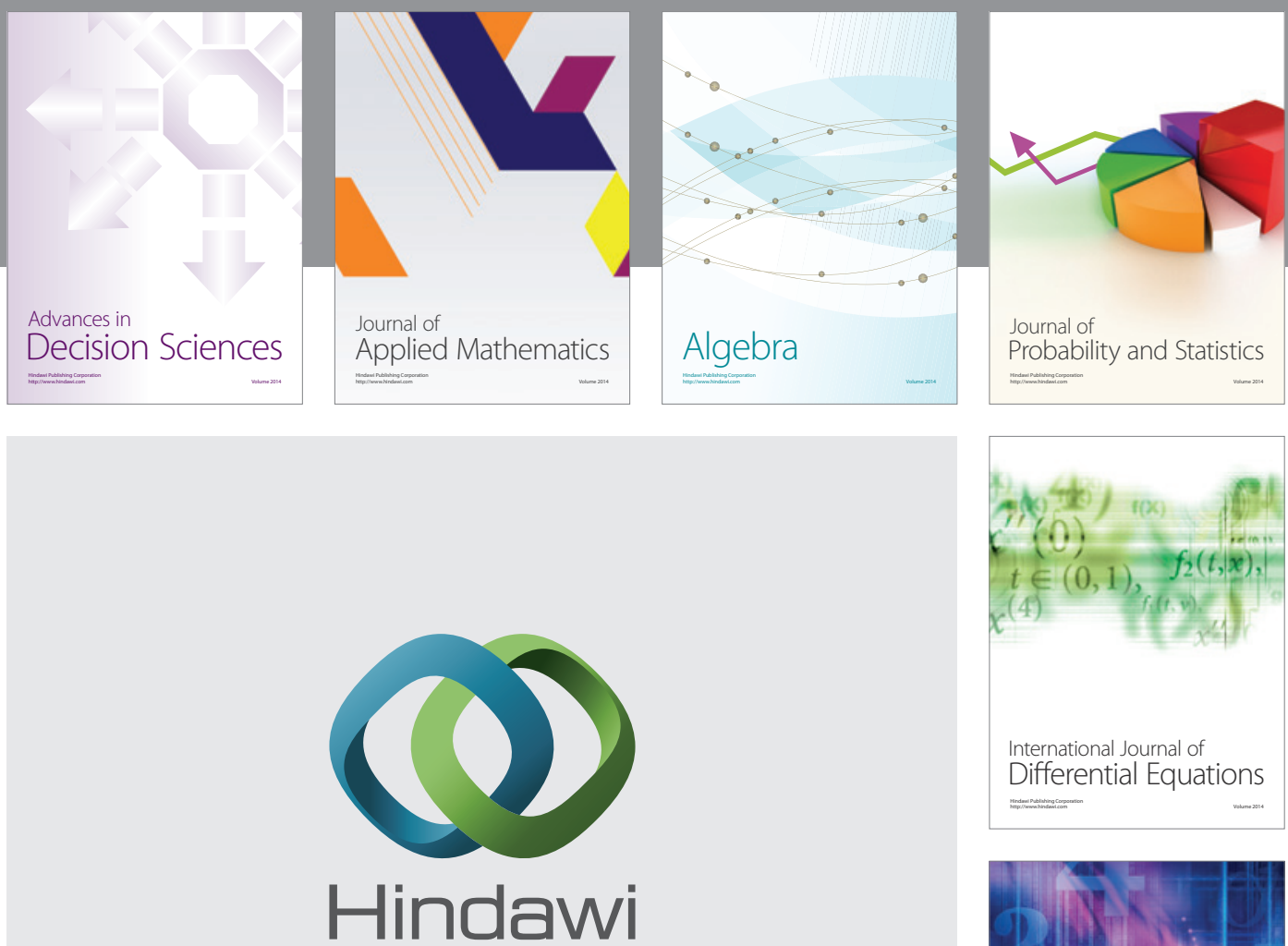

Submit your manuscripts at http://www.hindawi.com
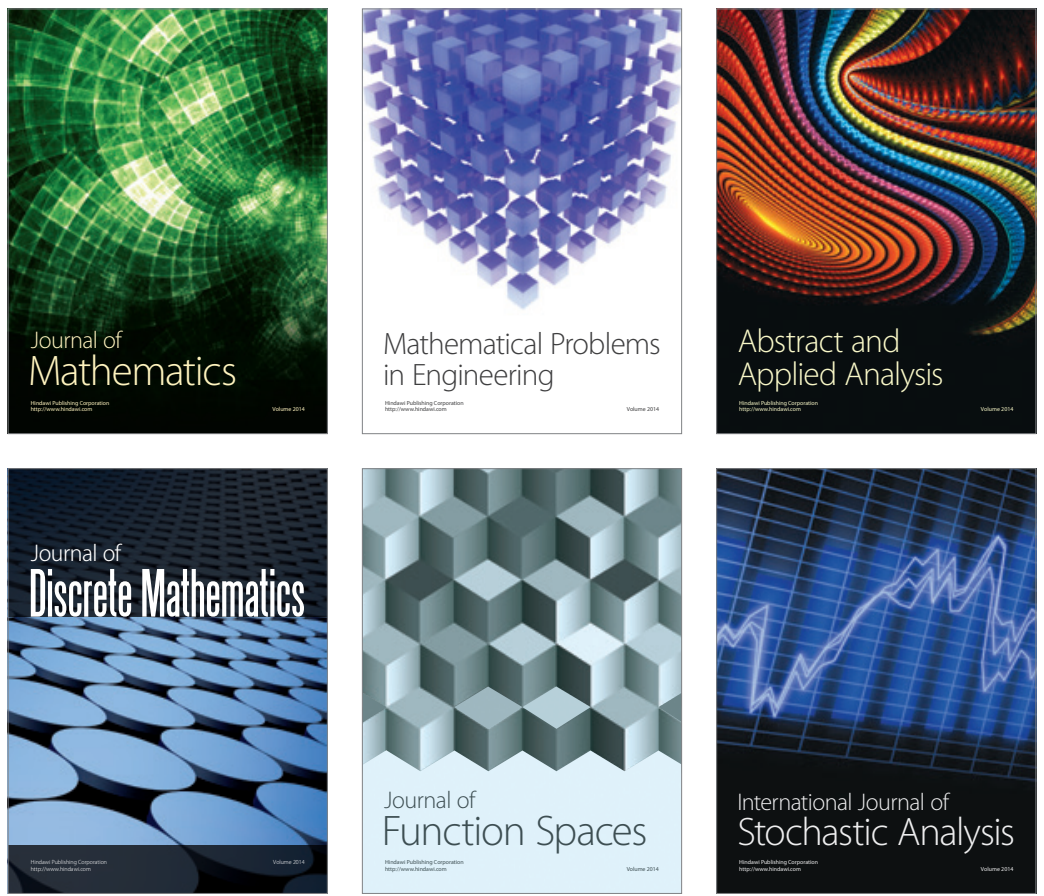

Journal of

Function Spaces

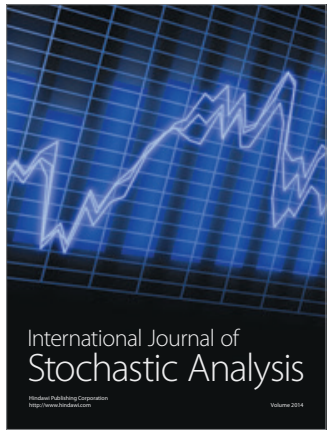

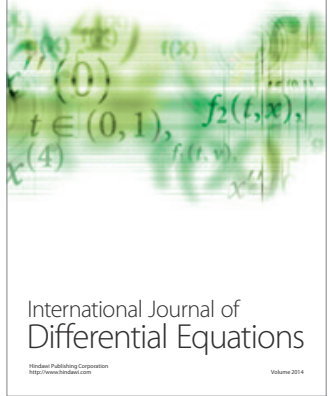
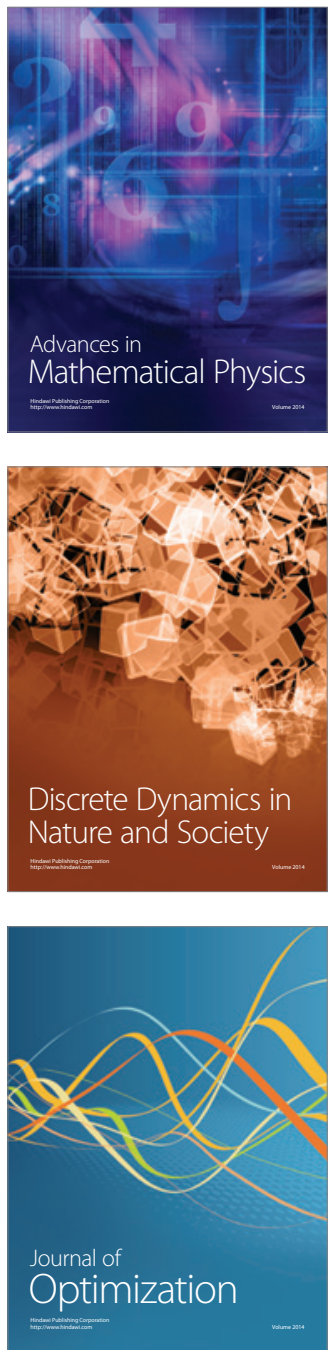